\title{
The Pathogenesis Based on the Glymphatic System, Diagnosis, and Treatment of Idiopathic Normal Pressure Hydrocephalus
}

This article was published in the following Dove Press journal:

Clinical Interventions in Aging

\author{
Changwu Tan ${ }^{1-3, *}$ \\ Xiaoqiang Wang ${ }^{4, *}$ \\ Yuchang Wang ${ }^{1-3}$ \\ Chuansen Wang ${ }^{1-3}$ \\ Zhi Tang ${ }^{5}$ \\ Zhiping Zhang ${ }^{1-3}$ \\ Jingping Liu ${ }^{1-3}$ \\ Gelei Xiao (iD) ${ }^{1-3}$ \\ 'Department of Neurosurgery, Xiangya \\ Hospital, Central South University, \\ Changsha, Hunan 410008, People's \\ Republic of China; ${ }^{2}$ Diagnosis and \\ Treatment Center for Hydrocephalus, \\ Xiangya Hospital, Central South \\ University, Changsha, Hunan 410008, \\ People's Republic of China; ${ }^{3}$ National \\ Clinical Research Center for Geriatric \\ Disorders, Xiangya Hospital, Central \\ South University, Changsha, Hunan \\ 4 10008, People's Republic of China; \\ ${ }^{4}$ Pediatric Neurological Disease Center, \\ Xinhua Hospital, Shanghai Jiaotong \\ University School of Medicine, Shanghai \\ 200092, People's Republic of China; \\ ${ }^{5}$ Department of Neurosurgery, Hunan \\ Cancer Hospital and the Affiliated \\ Cancer Hospital of Xiangya School of \\ Medicine, Central South University, \\ Changsha, Hunan 4I00I3, People's \\ Republic of China
}

*These authors contributed equally to this work

Correspondence: Gelei Xiao Department of Neurosurgery, Xiangya Hospital, Central South University, Changsha, Hunan 410008, People's

Republic of China

$\mathrm{Tel}+86137073$ I 4060

Email xiaogelei@csu.edu.cn

\begin{abstract}
Idiopathic normal pressure hydrocephalus (iNPH) is a rare neurological disorder with no clear prevalence factors and is a significant danger to the elderly. The intracranial glymphatic system is the internal environment that maintains brain survival and metabolism, and thus fluid exchange changes in the glymphatic system under various pathological conditions can provide important insights into the pathogenesis and differential diagnosis of many neurodegenerative diseases such as iNPH. iNPH can be diagnosed using a combination of clinical symptoms, imaging findings and history, and cerebrospinal fluid biomarkers due to the glymphatic system disorder. However, only few researchers have linked the two. Shunt surgery can improve the glymphatic system disorders in iNPH patients, and the surgical approach is determined using a combination of clinical diagnosis and trials. Therefore, we have composed this review to provide a future opportunity for elucidating the pathogenesis of iNPH based on the glymphatic system, and link the glymphatic system to the diagnosis and treatment of iNPH. The review will provide new insights into the medical research of iNPH.
\end{abstract}

Keywords: idiopathic normal pressure hydrocephalus, glymphatic system, pathogenesis, diagnostic methods, shunt surgery

\section{Idiopathic Normal Pressure Hydrocephalus}

Normal pressure hydrocephalus (NPH) is a treatable but difficult to diagnose neurological disorder, ${ }^{1}$ which was first proposed by Adams et al, (1965). The patients exhibit classic a triad of symptoms including gait and balance disorders, urinary incontinence, and cognitive impairment. ${ }^{2,3}$

Idiopathic normal pressure hydrocephalus (iNPH) is a kind of NPH with no clear cause, often occurring in the elderly, and imaging analysis indicates a normal cerebrospinal fluid (CSF) pressure and enlarged ventricle. ${ }^{3}$ A previous study reported that it can be relieved through early diagnosis and surgical intervention. ${ }^{4}$ However, it is difficult to diagnose and distinguish the disease because there is no specific cause of iNPH. Therefore, there is still some controversy over the specific classification of iNPH. In Europe and Japan, iNPH is considered as an independently diagnosed disease, whereas in the United States it is classified as a subtype of Alzheimer's disease (AD). Although the iNPH disease theoretically exhibits a triad of symptoms typified by cognitive impairment, urinary incontinence, and gait disturbance, in actual cases few patients have all three symptoms at the same 
time. Once misdiagnosis occurs, the patient fails to receive effective treatment, thereby causing aggravation over time. Therefore, we can improve the diagnostic efficiency and identify potential patients with iNPH by learning about the symptoms and diagnosis of iNPH, as well as exploring more efficient assessment scales and comparative disease analysis. This may allow patients to receive appropriate treatment as early as possible.

Generally, iNPH is a relatively rare disease, mostly occurring in the elderly. Martin-Laez et al, (2016) concluded that iNPH is an aging-related disease and its prevalence will exponentially increase with age in the future. ${ }^{5}$ Previous studies have reported that the prevalence is about $3.7 \%$ in people above 65 years of age and $5.9 \%$ in individuals over 80 years of age, sometimes even rising to $8.9 \%$ in some regions, which is about four times the prevalence (2.1\%) between 65 to 79 years of age. ${ }^{2,6,7}$ iNPH was first reported in 1989 at a frequency of $0.5 \%$ in the elderly people over 67 years of age. ${ }^{8}$ However, it was not strictly a prevalence estimate since the whole population was not screened. Subsequent studies conducted between 1986 and 2000 reported that the prevalence was $2.1 \%$ in elderly individuals over 70 years of age, while the latest study reported a prevalence rate of $3.7 \%$ in elderly people over 65 years of age, but both estimates have age limits. ${ }^{2}$ Some studies have also shown that the prevalence of iNPH is not associated with gender, perhaps because of the geographical and sample differences. ${ }^{7,9}$ However, most of the above mentioned data has limitations, such as being applicable within a specific region or the inclusion of iNPH patients is based on surrogate information like the International Classification of Diseases diagnosis code for iNPH, ${ }^{10}$ which may result in a lower prevalence. Therefore, the prevalence of iNPH and the diagnostic guidelines still need to be further elucidated.

Shunt surgery is the most common treatment method for individuals with iNPH because it can effectively alleviate the condition of current iNPH patients. ${ }^{11}$ However, the prognosis gradually deteriorates over time. ${ }^{12}$ Since the patients are relatively older and may have several underlying diseases, taking their clinical indications into consideration may aid in improving the prognosis and their quality of life.

\section{Glymphatic System in the Brain}

CSF is primarily produced by the choroid plexus in the lateral ventricles, and then it flows through the third and fourth ventricles into the subarachnoid compartment of the skull and spinal cord, where it is absorbed by venous blood from convex arachnoid granules. In addition, CSF is drained to the cervical lymph nodes along the olfactory nerve through the cribriform plate towards the lymphatic vessels of the nasal mucosa. ${ }^{13}$ The meningeal lymphatic vessels, which are located around the dural sinus, middle meningeal artery, and cribriform plate, can also drain CSF to the cervical lymph nodes. ${ }^{14} \mathrm{CSF}$ from the subarachnoid space is driven into the perivascular spaces of the cerebral arteries on the brain surface. It then flows to the brain parenchyma through the astrocyte aquaporin (AQP) thereby forming interstitial fluid, which in turn flows into the perivascular spaces of the venules. Finally, it can be eliminated via the blood circulation. Only three subtypes of AQP (AQP1, 4, and 9) have been found in the mammalian central nervous system (CNS). ${ }^{15}$ Previous studies have reported that AQP1 is generally expressed in capillary endothelial cells, but its expression is inhibited in brain capillary endothelial cells, thereby effectively separating CSF from blood and interstitial fluid. ${ }^{16,17}$ On the other hand, AQP4 is expressed and polarized in the astrocyte terminals of the pericapillary spaces of the brain capillaries, and communicates with the glymphatic system circulation between CSF and interstitial fluid. ${ }^{18,19}$ Therefore, a portion of CSF flows into the brain parenchyma from the pericapillary Virchow-Robin Space via the AQP4 system of blood-brain barrier astrocytes, thereby forming interstitial fluid. ${ }^{20-22}$ This process also regulates local cerebral blood flow in accordance with the glymphatic system pressure. $^{21}$

The previous view assumed that this was a unidirectional flow, ${ }^{23}$ but Yamada et al, (2016) found that cerebrospinal fluid does not only flow unidirectionally from the site of generation to absorption. ${ }^{24}$ This is because as it flows through the dural sheaths or lymphatics surrounding the cranial and spinal nerves and meninges, CSF is drained into the cervical lymphatics and is involved in the regulation of CSF levels, suggesting that multiple factors influence the intracranial glymphatic circulation. A previous study reported that fluid exchange changes in the glymphatic system under various pathological conditions can provide important insights into the pathogenesis and differential diagnosis of many neurodegenerative diseases, such as iNPH. ${ }^{22}$ Therefore, we have composed this review to discuss iNPH from the perspective of the glymphatic system with the overarching goal of providing new insights into the study of iNPH. 


\section{Glymphatic System and the Pathogenesis of iNPH}

Studies on the fluid exchange between the ventricles, subarachnoid space, perivascular Virchow-Robin Space, and glymphatic system of patients with iNPH are increasing. Ringstad et al, (2018) and Eide et al, (2019) compared the efficiency of glymphatic clearance in iNPH patients with that in normal subjects by injecting contrast media. ${ }^{25,26}$ The obtained results indicated that the efficiency of glymphatic clearance was significantly reduced in iNPH patients, ${ }^{25,26}$ suggesting that glymphatic system disorders may be one of the causes of the disease (Figure 1). ${ }^{25-28}$

\section{Dynamic Disorders of Glymphatic System}

It was previously thought that the driving force for CSF movement came from the lateral ventricular plexus, but the development of imaging techniques has led to the discovery that the lateral ventricular plexus is only part of the driving force. ${ }^{17,23}$ It also consists of cardiac-related ICP pulsations, which are associated with systole and

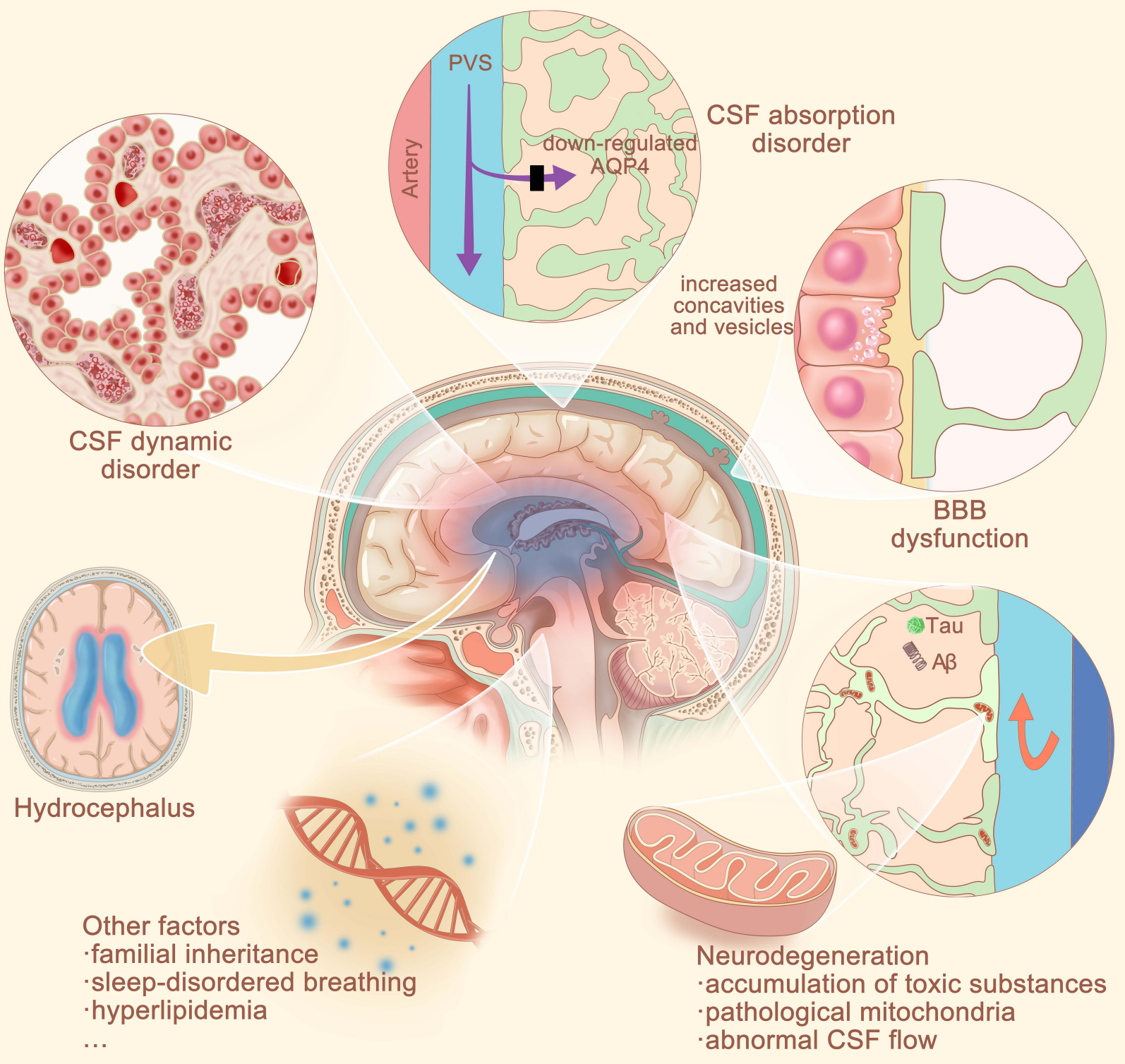

Figure I Pathogenesis of iNPH. Abnormalities in the choroid plexus pulses affected by heartbeat and respiratory rate, thereby leading to an impairment of CSF dynamics. Down-regulation of astrocyte terminal foot AQP4 protein, thereby leading to impaired CSF absorption. Abnormalities in the protein transport mechanism of blood-brain barrier endothelial cells, thereby leading to abnormal accumulation of proteins in the CSF. Impaired clearance and accumulation of neurotoxic substances, reduced transport efficiency of mitochondrial lesions, and abnormal flow of CSF, all resulting in neurodegenerative lesions. Other genetic, sleep apnea and hyperlipidemia factors may also be associated with the pathogenesis of iNPH.

Abbreviations: AQP, aquaporin; CSF, cerebrospinal fluid; iNPH, idiopathic normal pressure hydrocephalus. 
diastole of the heart. ${ }^{17}$ Schley et al, (2006) set up a theoretical model which demonstrated that the motive force is related to the pulse wave travelling along the arteries, and that a decrease in the pulse wave impedes glymphatic drainage within the brain. ${ }^{29}$ Several studies have reported that restricted cardiac activity leads to restricted arterial pulsations and decreased glymphatic flow, thereby resulting in disturbed CSF pulsation patterns and altered cerebral perfusion. ${ }^{22,27,30,31}$ Familiarly, respiratory-related ICP pulsations increases intracranial volume, decreases intracranial compliance, pressurizes cortical veins, and increases intraventricular pressure, ${ }^{32}$ which in turn leads to an imbalance of CSF production and absorption. ${ }^{33-35}$ In addition, respiratory and pathological intracranial pressure pulsations cause abnormal CSF flow and intracranial CSF redistribution. ${ }^{36}$ Previous related studies have found that the spinal CSF may be formed to buffer the increased demand for CSF flow through the lymphatic system during deep inspiration to compensate for venous outflow. ${ }^{36}$ Among them, the increase of CSF in the ventricles leads to its excessive accumulation in the suprasellar cistern, which may cause enlargement of the ventricles and put more pressure on the cortex. The ventricles would be further enlarged when the pressure exceeds the upper limit of cortical elastic tension, thereby causing the ICP to return to normal. ${ }^{3}$

\section{Absorption Disorders of the Glymphatic System}

The glymphatic system is absorbed into the peripheral veins of the brain, mainly through arachnoid granules. Generally, the blood-brain barrier, composed of tightly connected capillary endothelial cells in the brain, separates CSF from blood and restricts the exchange of macromolecules to maintain the osmotic pressure of CSF.

The main mediators connecting the CSF to the blood circulation are the AQP4 of the astrocytes surrounding the intracranial capillaries. Previous studies have found that iNPH patients have a decreased astrocyte AQP4 expression. $^{37,38}$ This can interfere with the homeostasis of the glymphatic system in the brain, or cause an imbalance in the supply and demand of CSF in the cortical capillaries and local subarachnoid space, thereby resulting in super-posterior displacement of brain tissues, which in turn leads to local ischemia. ${ }^{39}$ It is worth noting that the local ischemia is accompanied by disturbed neuronal metabolism and accumulation of neurotoxic substances in the glymphatics. ${ }^{16,40}$ A previous study reported that the accumulation of neurotoxic substances interferes with the stability of CSF perfusion. ${ }^{30}$ Furthermore, cerebral ischemia occurs concurrently with hypoxia, which leads to significant changes in the extracellular matrix composition, endosomal lysosomal systems, and mitochondrial energy metabolic capacity. ${ }^{41}$ This results in an imbalance in the supply of ions and metabolites, and abnormal circulation in the glymphatic system of the brain, ultimately leading to neurodegeneration. ${ }^{30}$

For example, deletion of the AQP4 gene decreases the clearance of cerebral interstitial fluid through the periventricular Virchow-Robin Space drainage pathway by approximately $70 \%$, thereby leading to abnormal CSF flow and intermediate metabolite disruption, which ultimately leads to slow neurodegeneration. ${ }^{37}$ Moreover, the mitochondria-endoplasmic reticulum contact sites regulate the metabolism of soluble amyloid $\beta(\mathrm{A} \beta)$, whose number is positively correlated with age in iNPH patients. ${ }^{42}$ Hasan-Olive et al, (2019) found pathological mitochondria in patients, ${ }^{43}$ whose presence may also contribute to the deposition of soluble $A \beta$ and cause mental disorders in iNPH patients. In addition to impaired uptake by the glymphatic system, several other studies have found that patients with iNPH also partially exhibit reduced corticospinal excitability and reduced intracortical inhibitory connections in frontal and primary motor areas. ${ }^{44-46}$ This finding suggests that neurotransmitters such as $\gamma$ aminobutyric acidergic and cholinergic neurotransmitters are also affected. ${ }^{44-46}$

In addition to the AQP4 system, both blood-brain barrier protein leakage and impaired circulation of CSF may lead to abnormal impairment of glymphatic metabolism, thereby causing sub-ischemia which ultimately leads to glial hyperplasia and neuronal degeneration, ${ }^{47}$ exhibiting possible manifestations of iNPH. Ueno et al, (2006) examined blood-brain barrier permeability in a mice model of neural cell-specific hypoxia-inducible factor- $1 \alpha$ deficient-induced hydrocephalus. ${ }^{48}$ They found no significant difference between the mice model and the controls, suggesting that spontaneous hydrocephalus may not, in turn, significantly affect the blood-brain barrier. ${ }^{48}$ In a study of tissue biopsies obtained from iNPH patients, Andreone et al, (2017) reported that the patients did not have severe damage to the small blood vessels in the brain, but rather an increase in vesicles in the luminal side of the capillary endothelial cell lumen and in the cytoplasm. ${ }^{49}$ The vesicles are loaded with blood proteins including 
fibronectin (pro), which play a transmembrane role, and thus their abnormal increase can lead to leakage of blood proteins. $^{49}$ Another study reported that large amounts of CSF entering the bloodstream also appear to pass through the large vesicles formed on the luminal surface of endothelial cells, where they discharge their contents into the venous bloodstream. ${ }^{50}$ Hasan-Olive et al, (2019) also found that the presence of pathological mitochondria in perivascular astrocytes in patients' cerebral vessels severely affects transmembrane protein transport. ${ }^{43}$ The dysfunction of neurovascular units in the blood-brain barrier may also lead to neurodegenerative changes characterized by iNPH, failure of para-vascular clearance, and CSF circulation disorders. ${ }^{51}$

Satow et al, (2017) reported that cerebral deep vein dysfunction in iNPH patients leads to a difference in drainage capacity between the superficial and deep venous systems, which in turn results in a transmembrane pressure in the brain parenchyma glymphatic system that accelerates the disease process. ${ }^{52}$ In addition, basement membrane and pericytes also maintain the stability of the blood-brain barrier, suggesting that they are also involved in the development of hydrocephalus to some extent. ${ }^{53,54}$

\section{Glymphatic System and Diagnosis of iNPH}

iNPH is a chronic disease that undergoes different factors leading to ventricular dilation and white matter lesions, thereby creating a vicious circle that further produces a triad of symptoms typified by cognitive impairment, urinary incontinence, and gait disturbance. According to international and Japanese guidelines, the diagnosis of iNPH relies on a combination of brain imaging and clinical features, with history taking also contributing to the diagnosis (Figure 2). ${ }^{55,56}$ However, in practice, iNPH patients have often sought therapeutic help from multiple specialists (psychiatry, urology, and internal medicine) due to the diversity of clinical symptoms and the difficulty of differential diagnosis from other neurological disorders. Therefore, how to effectively improve the efficiency of early diagnosis and treatment of iNPH is still one of the hot research topics. Perhaps an improved diagnostic approach based on the glymphatic system would be a useful approach.

\section{Clinical Manifestation}

It is vital to focus on the clinical manifestations before making a definite diagnosis of iNPH. Therefore, patients who are clinically compatible with iNPH should be included for further imaging or glymphatic function testing. However, we cannot exclude the effect of glymphatic dysfunction on the clinical presentation and imaging of iNPH, despite there being no more data to support the association. Several studies have reported that intracranial disorders of the glymphatic system may result in loss of homeostasis of the neuronal environment, which may contribute to neuronal malfunction and compression of parts of the cerebral cortex. ${ }^{28,57,58}$ In the case of iNPH, disorders of the glymphatic system caused by different factors can create ventricular dilation and even cerebral white matter lesions, thereby further producing a triad typically characterized by cognitive impairment, urinary incontinence, and gait and balance disorders. It is worth noting that gait and balance disorders are the main clinical manifestations of iNPH patients, while cognitive impairment and urinary incontinence occur as the disease progresses. ${ }^{59}$

\section{Gait and Balance Disorders}

Gait impairment in iNPH is best characterized as a higherlevel gait disorder. It involves difficulty integrating sensory information about the body's position in its environment in the absence of primary sensorimotor deficits, cerebellar dysfunction, or involuntary movements. ${ }^{60}$ It also includes the effects of gravity, and correctly selecting and executing movement plans for gait or postural reflexes. ${ }^{60}$ These are demonstrated by retropulsion or anteropulsion of stance, hesitation or failure to initiate gait, shuffling and widebased gait, reduced foot clearance, and difficulty with turning. ${ }^{55,56,61,62}$ Selge et al, (2018) and Nikaido et al, (2018) found that patients with iNPH have a $60-80 \%$ probability of falling due to gait and balance disorders. ${ }^{62-64}$ Parkinson's disease (PD), myasthenia gravis, and osteoarthritis (diseases that can also be caused by intracerebral glymphatic system dysfunction), can also cause gait disorders and thus they should be excluded during the diagnostic process. Assessment methods for gait and balance disorders, include but not limited to: timed Up and Go, 10-meter walk test, Functional Gait Assessment, and Berg Balance Scale. $^{65-68}$

\section{Cognitive Impairment}

The cognitive impairment in iNPH patients manifests as frontal-to-subcortical dementia characterized by executive dysfunction, psychomotor slowing, and mood symptoms, especially apathy. ${ }^{56,69,70}$ Approximately $78-98 \%$ of patients with iNPH have cognitive impairment, with the 


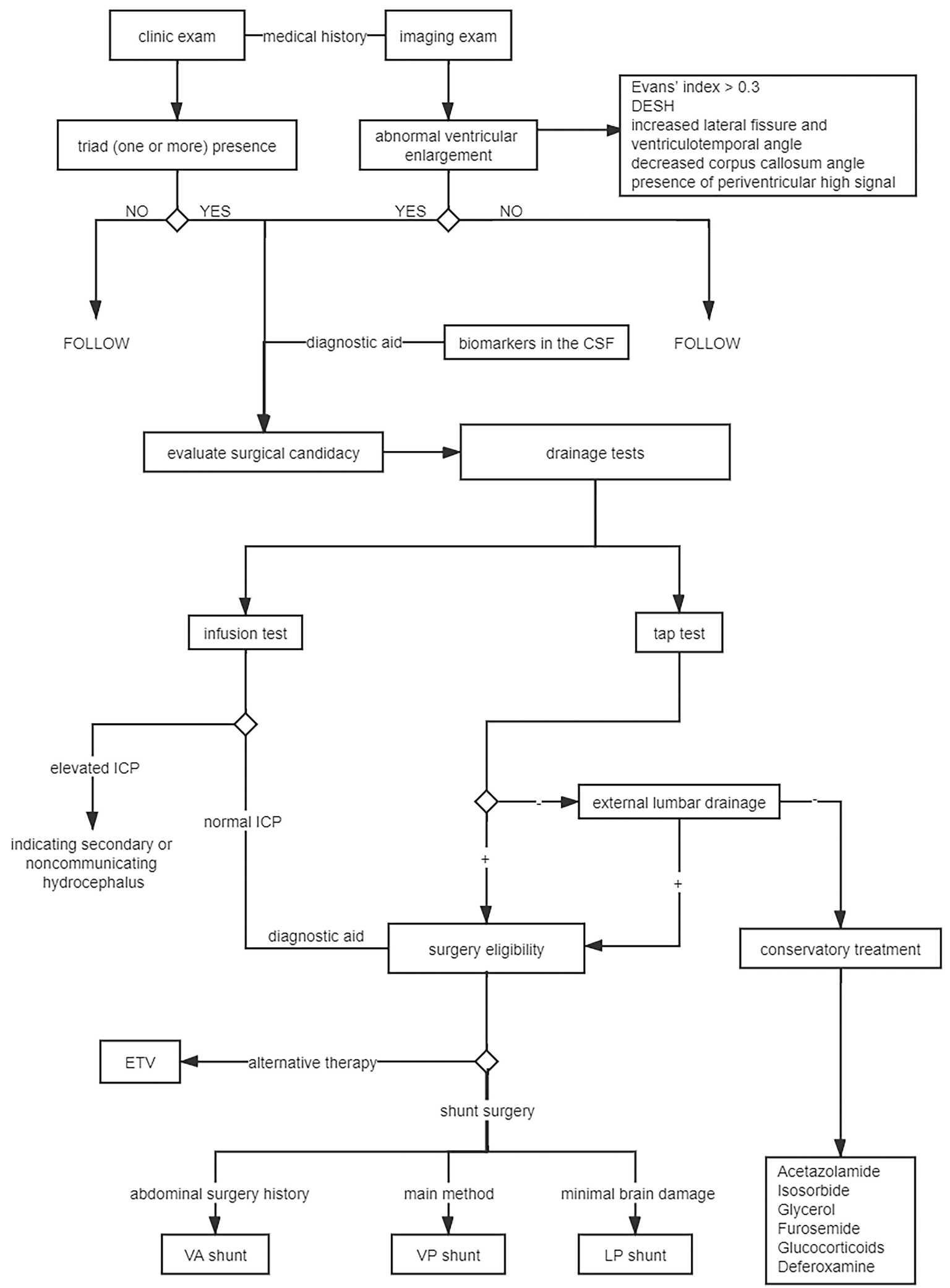

Figure 2 Flowchart for diagnosis and treatment of iNPH patients. The clinical diagnosis of a patient with at least one of the triad of symptoms, together with the imaging diagnosis of abnormal ventricular enlargement, can raise suspicion of iNPH. Medical history and cerebrospinal fluid biomarkers can also assist in the diagnosis. A positive TT can indicate that the patient is eligible for surgery, while a negative TT indicates that the patient requires further testing through an ELD. On the other hand, a positive ELD also indicates that the patient is eligible for surgery, while a negative ELD indicates that the patient requires conservative treatment. In addition, infusion testing can assist in measuring the intracranial pressure to determine the eligibility for surgery. Surgery is most often performed using shunt surgery, and ETV is an alternative surgical therapy.

Abbreviations: CSF, cerebrospinal fluid; DESH, disproportionately enlarged subarachnoid-space hydrocephalus; ELD, external lumbar drainage; ETV, endoscopic third ventriculostomy; ICP, intracranial pressure; LP shunt, lumboperitoneal shunt; TT, tap test; VA shunt, ventricular atrial shunt; VP shunt, ventriculoperitoneal shunt. 
degree varying from mild cognitive impairment to dementia. ${ }^{55}$ During clinical diagnosis, frontal assessment battery, $^{71}$ the Mini-Mental State Examination, ${ }^{72}$ and Montreal cognitive assessment ${ }^{73}$ are often used to assess the cognitive function of patients. In practice, Lewy body dementia, PD, and corticobasal syndrome can also produce cognitive dysfunction similar to iNPH. Therefore, the diagnosis should be identified using a combination of neuropsychological examination and fluid drainage tests that can affect the intracranial glymphatic system. ${ }^{69}$ Care also needs to be taken to exclude the effects of schizophrenia, which can have a significant negative impact on cognition, and the inability to obtain a valid evaluation of cognitive function. ${ }^{55,56}$

\section{Urinary Incontinence}

Approximately $76-83 \%$ of patients with iNPH typically present with urinary incontinence characterized by urgency and difficulty, which inhibits bladder emptying. ${ }^{55,74}$ Urinary incontinence due to involuntary urination may represent the presence of other comorbidities, and thus the patient's urinary function requires a thorough examination to rule out other neurogenic or urologic disorders, including but not limited to prostate disease and bladder dysfunction.

\section{Drainage Test}

Diseases caused by dysfunction of the glymphatic system can also be diagnosed by interfering with the abnormal glymphatic system and observing changes in the clinical presentation. Drainage tests are the most commonly used test for patients with suspected iNPH, ${ }^{56,75}$ with the tap test (TT), external lumbar drainage (ELD), and infusion testing being the three commonly used tests.

\section{Tap Test}

Tap test (TT) involves performing a standard lumbar puncture to remove a large volume of CSF from the lumbar CSF space, which is also associated with the glymphatic system. $^{55}$ The reliability of shunt surgery is often estimated by comparing the gait assessment before and after TT because of the better improvement of gait disorder by shunt surgery, and the assessment process can yield good results with or without the aid of equipment. ${ }^{76,77}$ Most importantly, a sufficient volume of CSF needs to be drained to have an effect on the glymphatic system function, which can improve symptoms over time, in order to better assess the shunt effect. ${ }^{56,59}$ Gait is usually reassessed $30 \mathrm{~min}$ to $4 \mathrm{~h}$ after the test, and there is also an assessment of urinary and cognitive function after one week in clinical trials. ${ }^{71,78}$ In addition, TT can also be used to distinguish between normal aging-induced and iNPHinduced cerebral deep vein dysfunction, due to the fact that the latter can be rectified in iNPH patients after TT, whereas normal aging cannot. ${ }^{52}$ A previous study reported that the duration of the disease is also a factor, and early detection in the course of the disease yields a high degree of accuracy. ${ }^{79}$ Furthermore, a recent study found that the use of TT to assess the applause sign can effectively delineate the subtype of frontosubcortical disorders in iNPH patients. $^{80}$ The positive predictive value (PPV), negative predictive value (NPV), and accuracy of TT for a favorable response to shunting are approximately $73-100 \%, 16-42 \%$, and $45-54 \%$, respectively. ${ }^{55,81}$

\section{External Lumbar Drainage}

External lumbar drainage (ELD) requires the patient to be hospitalized and undergo continuous CSF drainage, often performed when symptoms do not improve after TT and iNPH is still suspected. ${ }^{81}$ Briefly, a tuohy needle is inserted and CSF is drained at a slower rate for long periods of time in large volumes $(10-14 \mathrm{~mL} / \mathrm{h}$ for about $72 \mathrm{~h}) .{ }^{55,56}$ Moreover, gait evaluation is still required after drainage. ELD is a more reliable method for predicting shunt response in iNPH than TT, with its PPV, NPV, and accuracy values being approximately $1-100 \%, 36-100 \%$, and $58-100 \%$, respectively. ${ }^{81}$ However, it requires a more specialized physician to perform, and is not the preferred clinical choice due to its complexity and the potential for infection from prolonged drainage.

\section{Infusion Test}

The infusion test is a "quick, easy, and handy" diagnostic protocol which determines the CSF outflow resistance or CSF conductance. However, it is less commonly used worldwide, and is only common in Europe. Briefly, two lumbar puncture needles are inserted into the lumbar subarachnoid space: the first needle is connected to an infusion pump to infuse artificial CSF, while the second needle is connected to a closed-pressure recording device. ${ }^{82}$ Previous studies have reported that the inclusion of CSF outflow resistance values in the selection of patients undergoing shunt surgery has been associated with a significant improvement in postoperative patient outcomes. ${ }^{83,84}$ The test also requires specialized physicians and specialized 
equipment to perform, and has a PPV of approximately $75-92 \% .{ }^{55}$

\section{Biomarkers in the Glymphatic System}

iNPH is a multifactorial disease with a high number of neuropathological changes, which in turn result in changes in the composition of the glymphatic system such as CSF, despite the absence of specific signs. CSF protein biomarkers reflect fundamental pathological changes occurring in the CNS and the changes may have positive implications for the pathophysiology of iNPH pathogenesis. ${ }^{85}$ Therefore, these changes are of great significance for the early diagnosis of iNPH because they partially trend in the early stages of the disease. However, they need to be differentiated from other neurological diseases and thus more research is required to explore them.

Schirinzi et al, (2018) found that the markers of subcortical damage (neurofilament light, myelin basic protein, and leucine-rich- $\alpha 2$-glycoprotein) were increased in iNPH patients. ${ }^{85}$ Other significant findings included the reduction of all amyloid-beta-related proteins, either soluble amyloid precursor proteins or $\mathrm{A} \beta$ fragments $(\mathrm{A} \beta 42, \mathrm{~A} \beta 38$, and $\mathrm{A} \beta 40)$, together with the mild lowering of tau proteins (total tau and phosphorylated tau). ${ }^{85-87}$ In combination with these findings, elevated monocyte chemoattractant protein 1 was shown to separate patients with iNPH from healthy individuals and patients with vascular dementia, PD, multiple system atrophy, progressive supranuclear palsy, corticobasal degeneration, $\mathrm{AD}$, frontotemporal lobe degeneration, and other cognitive and movement disorders with a good diagnostic sensitivity and specificity. ${ }^{88}$ The $A \beta 42 / A \beta 40$ ratio also contributes to the differential diagnosis of $\mathrm{NPH}$ with $\mathrm{AD}$ and other dementias, but shows poor associations with clinical variables. ${ }^{89}$

Although CSF biomarkers have been studied extensively, the study of CSF biomarkers in iNPH is relatively new. Thus, iNPH-specific CSF biomarkers with strong evidence have not yet been identified, and there is no proven or substantiated role in diagnosing iNPH. However, research methods continue to innovate and provide new ideas for the exploration of CSF biomarkers and the diagnosis of iNPH such as two-point CSF collection and observing dynamic changes in CSF biomarkers, ${ }^{90}$ which is providing new ideas for auxiliary diagnostics.

\section{Radiological Examination}

Currently, the connection between imaging and the glymphatic system has not been precisely defined, although many researchers have attempted to visualize the function and structure of the glymphatic system in vivo using MRI. ${ }^{91}$ Several scientists have used the "Diffusion Tensor Image-Analysis along the Perivascular Space" method to examine iNPH patients. It has been found to be a useful imaging tool for identifying glymphatic dysfunction and for individually quantifying the glymphatic activity in iNPH patients. ${ }^{92,93}$ Disorders of the glymphatic system in the brain can affect different parts of the brain, thereby manifesting different diseases, some of which can be distinguished by neuroimaging. For example, a previous study reported that diffusion tensor imaging provides a quantitative measure of neurological fractional anisotropy (FA).$^{94} \mathrm{FA}$ decreases in general neurological diseases but increases in the corticospinal tract in iNPH patients, a phenomenon that distinguishes iNPH from other neurological diseases such as AD and PD. ${ }^{94-96}$

In addition, visualization of brain lesions can be represented by imaging and is an important diagnostic modality in tradition. The most common diagnostic imaging marker in iNPH patients is the abnormal enlargement of the ventricles. Patients with iNPH present with abnormally enlarged ventricles without CSF flow disorders, but those with enlarged ventricles do not necessarily have iNPH. ${ }^{56}$ The main indicators for evaluating ventricular enlargement are Evans' index, callosal angle, the size of temporal horns, narrow high-convexity sulci, dilated Sylvian fissures, focally dilated sulci, periventricular hypodensities, and bulging of the lateral ventricular roof. ${ }^{1,97,98}$ Evans' index, the ratio of the widest frontal horn span to the widest diameter of the brain on the same axial image, is a more frequently used test. However, both the imaging angle and the layer affect the EI value, and the value is age-dependent. Patients with iNPH exhibit a positive Evans' index $(>0.3),{ }^{99-101}$ but it is not specific for patients with brain atrophy due to other factors such as $\mathrm{AD} .{ }^{100}$ A recent study presented a new method for assessing the anteroposterior diameter of the lateral ventricle index $(>0.50)$, which can distinguish iNPH patients and agematched elderly subjects with healthy Evans' index. ${ }^{102}$

Some other imaging manifestations may also help in the diagnosis of iNPH such as disproportionately enlarged subarachnoid-space hydrocephalus (DESH), which is also a form of glymphatic dysfunction. ${ }^{103}$ Several studies have shown a good correlation between iNPH and DESH signs, ${ }^{39,103-105}$ and the combination of DESH features measured using voxel-based morphometry and the Evans' index can distinguish $\mathrm{iNPH}, \mathrm{AD}$, and normal 
aging. ${ }^{106}$ However, Takagi et al, (2020) reported that the Evans' index $>3.0$ and DESH lead to a $70 \%$ misdiagnosis rate when both are included in the diagnostic criteria for iNPH. ${ }^{107}$ Therefore, DESH is not yet specific for judging whether a patient has iNPH, and it is still highly controversial in the available studies.

\section{Glymphatic System and Treatment of iNPH}

In general, iNPH is a progressive neurological disorder, and glymphatic disorders can continue to cause damage to the brain if left untreated. ${ }^{108}$ Therefore, patients generally undergo surgery as soon as possible after the diagnosis of iNPH, including Ventriculoperitoneal shunt (VP shunt), Ventricular atrial shunt (VA shunt) and Lumboperitoneal shunt (LP shunt), and alternative surgery such as endoscopic third ventriculostomy (ETV). The appropriate surgical treatment is often decided based on the patient's specific clinical assessment and medical history. Isik et al, (2019) reported that the deterioration of gait disturbance may be improved, and cognitive decline may be stabilized by applying recurrent CSF removal. ${ }^{68}$ However, patients who are not candidates for surgery will be treated medically and conservatively due to the complications of shunt surgery, and the patient's age and physical condition (Figure 2).

\section{Conservative Treatment}

Conservative treatment is often symptomatic and based on the patient's clinical presentation, and it alleviates the patient's temporary symptoms which cannot be thoroughly cured. A previous study proposed acetazolamide, a carbonic anhydrase inhibitor, as an alternative to shunting for adults with NPH in order to address glymphatic dysfunction in the brain. ${ }^{109}$ The choroid plexus contains high levels of carbonic anhydrase and acetazolamide effectively inhibits the production of choroid plexus CSF. In addition, acetazolamide has been shown to inhibit aquaporin-mediated water conductance through several molecular pathways. ${ }^{110}$ In addition, Alperin et al, (2014) reported that administration of oral acetazolamide is associated with a decrease in the periventricular hyperintensities observed through MRI. ${ }^{111}$ Other drugs including isosorbide, glycerol, furosemide, glucocorticoids, and deferoxamine, can also be used for conservative treatment of hydrocephalus, ${ }^{112}$ but no corresponding studies are available for iNPH treatment.

\section{Shunt Surgery}

According to clinical guidelines, early shunt surgery is still recommended for patients with iNPH to improve intracranial glymphatic dysfunction. ${ }^{11}$ The indication for surgery is determined using the sum of clinical symptom data (complete or incomplete triad), hydrocephalus images obtained by MRI or $\mathrm{CT}$, and test results (a positive test result is an absolute indication for surgical treatment). ${ }^{113}$ A shunt system is comprised of three connected parts: a proximal catheter which may be placed in the lateral ventricle or lumbar cistern; a distal catheter which is placed in an absorptive cavity such as the peritoneum or the atrium of the heart; and a valve which connects the proximal and distal catheters to help regulate the rate of CSF drainage. ${ }^{55}$ Some studies found that the CSF biomarkers were altered after undergoing shunt surgery, suggesting that shunt surgery may improve glymphatic system dysfunction. ${ }^{114}$

\section{Ventriculoperitoneal Shunt}

The current standard treatment for iNPH involves the implantation of a ventriculoperitoneal (VP) shunt. ${ }^{115}$ Several studies have demonstrated improvements in cognition, gait, and urinary function in iNPH patients after VP shunt; ${ }^{75,116}$ with an average improvement of approximately $71 \%{ }^{117}$ Glymphatic factors that predicted a positive outcome with VP shunt include higher CSF outflow resistance, a positive response to an ELD or TT, and a positive reduction in cerebral blood flow with the use of acetazolamide. ${ }^{75}$ On the other hand, the factors that predicted a negative outcome included higher age and previous cerebrovascular disease. ${ }^{118}$ Common early complications of the surgery include infection, subdural hematoma and subdural fluid, and occurrence of occasional excessive drainage. ${ }^{119}$ However, the improvements in shunt technology and shunt devices such as the use of anti-siphon devices and the selection of shunt valve pressure have resulted in the complications being avoided. Farahmand et al, (2016) reported that there is no clinically significant difference between using $40 \mathrm{mmH}_{2} \mathrm{O}$ and 200 $\mathrm{mmH}_{2} \mathrm{O}$ in shunt valve pressure. ${ }^{120}$ Moreover, a personalized dynamic pressure strategy can be developed based on the patient's clinical manifestations, imaging results, and changes in the drainage test. The use of an adjustable valve gate can effectively aid in the realization of this protocol. ${ }^{121}$

\section{Ventricular Atrial Shunt}

Ventricular atrial shunt (VA) shunt is the main treatment option for the patients who are not suitable for VP shunt 
due to some abdominal factors ${ }^{122}$ such as ascites, overweight, or history of abdominal surgery. ${ }^{123,124}$ NunooMensah et al, (2009) reported that approximately $43.8 \%$ of the patients over the age of 65 have undergone abdominal surgery. ${ }^{125}$ Previous studies have also reported that there is no significant difference in gait improvement between patients who underwent VA shunt and those that underwent VP shunt, and the overall incidence of postoperative complications (infection and shunt obstruction) is similar in both groups. ${ }^{12,126}$ Subdural hematoma is the most common complication in patients after VA and VP shunt, but Mcgovern et al, (2014) reported that its incidence is significantly higher after VA shunt than after VP shunt. ${ }^{126}$ In addition, VA shunt may cause cardiopulmonary complications such as endocarditis and pulmonary heart disease, thereby leading to increased post-morbid mortality. ${ }^{12}$ Thus, VA shunt surgery is often an alternative treatment method despite being an effective modality for the treatment of iNPH. Therefore, VA shunt may be more widely used if a safe and favorable condition is provided.

\section{Lumboperitoneal Shunt}

Lumboperitoneal (LP) shunt is a minimally invasive procedure which does not cause direct trauma to the brain, thereby avoiding the development of hematomas in the brain parenchyma. ${ }^{34}$ A study comparing LP and VP shunts found that both improved symptoms in patients with possible INPH, with no significant difference in outcomes between the two methods, ${ }^{115}$ and no significant difference in the incidence of postoperative complications. ${ }^{127}$ In Japan, LP shunt is used for the treatment of iNPH in a larger number of patients despite not being widely used internationally. ${ }^{128}$ This is because it is the least invasive treatment method and it causes the least damage to the brain.

In addition to the above mentioned three types of shunts, we also have ventriculopleural shunt, retrograde ventriculosinus shunt, ${ }^{129}$ and ventriculogallbladder shunt, ${ }^{57}$ but there is a lack of strong data to prove their safety and efficacy. Moreover, there are currently no international standards for evaluating the results of various types of surgeries, and the improvement rates of patients after surgery are partially different. In some cases, extra shunt surgery is required when catheters are misplaced or infections occur aggravating disorders of the glymphatic system. A previous study involving a 12-month follow-up survey reported that $14.7 \%$ of patients required extra shunt surgery. ${ }^{119}$

\section{Endoscopic Third Ventriculostomy}

Endoscopic third ventriculostomy (ETV) is the use of a neuroendoscope to puncture a hole in the bottom of the third ventricle with the goal of establishing a channel between the ventricular system and the subarachnoid space, which permits CSF to flow into the suprasellar cistern. This approach directly alters the circulatory pathways of the glymphatic system and, in clinical trials, its overall benefits outweigh the disadvantages. Moreover, ETV is also a promising treatment option for iNPH in cases where there is evidence of narrowing of the fourth ventricular outflow tract catheter. ${ }^{130,131}$ ETV is mostly used to treat obstructive hydrocephalus, but many trials have started using it to treat iNPH. ${ }^{132,133}$ The average success rate of ETV for the treatment of iNPH is reported to be as high as $63 \%$, but more than $50 \%$ of patients require revision surgery after ETV. Therefore, it is not the primary treatment method for patients with iNPH and there is no proven or substantiated role. ${ }^{121}$

\section{Conclusions and Prospective}

iNPH is one of the few neurodegenerative diseases that can be treated successfully, but, in reality, it is still a severe disease in many patients. Physicians make a variety of diagnoses based on clinical guidelines due to the variability of patients' conditions. Nevertheless, misdiagnosis and omission still occur, which leads to further deterioration of patients. In-depth investigation of the links between the glymphatic system and the pathogenesis of iNPH can optimize the current diagnostic approaches and prevent early aggravation of the disease. Furthermore, studies can identify the characteristics of the disease with a high degree of correlation by targeting the differential expression of neuroimaging and CSF biomarkers in patients with iNPH. A separate exploration of each component is not sufficient for gaining a deep understanding of iNPH, and thus combining the components and setting up different weighted scales may be more helpful in the diagnosis of iNPH. In response to the increased glymphatic volume in the brain, iNPH patients are often advised to undergo shunt surgery as soon as possible after diagnosis. The adjustable-valve gate in shunt surgery reduces the incidence of post-shunt complications, thereby resulting in a better prognosis for patients after one year. However, the prognosis gets worse over time. ETV can also be widely-used as a therapeutic means of treating iNPH if more robust studies demonstrate the 
effectiveness of ETV in iNPH patients. In addition, recurrent CSF removal may be an alternative treatment approach for the patients who are not candidates for surgery. However, further studies should be conducted in the future to determine the factors influencing positive and negative shunt results, as well as diagnostic screening and improvement of shunt and ETV in patients with iNPH, with the overarching goal of giving superior outcomes for iNPH patients.

\section{Abbreviations}

$\mathrm{A} \beta$, amyloid $\beta ; \mathrm{AD}$, Alzheimer's disease; $\mathrm{AQP}$, aquaporin; CNS, central nervous system; CSF, cerebrospinal fluid; CT, computed tomography; DESH, disproportionately enlarged subarachnoid-space hydrocephalus; ELD, external lumbar drainage; ETV, endoscopic third ventriculostomy; FA, fractional anisotropy; ICP, intracranial pressure; iNPH, idiopathic normal pressure hydrocephalus; LP shunt, lumboperitoneal shunt; MRI, magnetic resonance imaging; NPH, normal pressure hydrocephalus; NPV, negative predictive value; PD, Parkinson's disease; PPV, positive predictive value; TT, tap test; VA shunt, ventricular atrial shunt; VP shunt, ventriculoperitoneal shunt.

\section{Acknowledgments}

This work was supported by Hunan Provincial Natural Science Foundation of China (No. 2019JJ50949) and a grant from the research projects of Hunan Provincial Health Commission of China (No. B2019187).

\section{Disclosure}

The authors report no conflicts of interest in this work.

\section{References}

1. Andersson J, Rosell M, Kockum K, Soderstrom L, Laurell K. Challenges in diagnosing normal pressure hydrocephalus: evaluation of the diagnostic guidelines. e Neurological Sci. 2017;7:27-31. doi:10.1016/j.ensci.2017.04.002

2. Zaccaria V, Bacigalupo I, Gervasi G, et al. A systematic review on the epidemiology of normal pressure hydrocephalus. Acta Neurol Scand. 2019;141(2):101-114.

3. Hakim S, Adams RD. The special clinical problem of symptomatic hydrocephalus with normal cerebrospinal fluid pressure. Observations on cerebrospinal fluid hydrodynamics. J Neurol Sci. 1965;2 (4):307-327.

4. Rekate HL, Blitz AM. Hydrocephalus in children. Handb Clin Neurol. 2016;136:1261-1273.

5. Martin-Laez R, Caballero-Arzapalo H, Valle-San Roman N, LopezMenendez LA, Arango-Lasprilla JC, Vazquez-Barquero A. Incidence of idiopathic normal-pressure hydrocephalus in Northern Spain. World Neurosurg. 2016;87:298-310.
6. Andersson J, Rosell M, Kockum K, Lilja-Lund O, Soderstrom L, Laurell K. Prevalence of idiopathic normal pressure hydrocephalus: A prospective, population-based study. PLoS One. 2019;14 (5):e0217705.

7. Jaraj D, Rabiei K, Marlow T, Jensen C, Skoog I, Wikkelso C. Prevalence of idiopathic normal-pressure hydrocephalus. Neurology. 2014;82(16):1449-1454.

8. Casmiro M, Benassi G, Cacciatore FM, D'Alessandro R. Frequency of idiopathic normal pressure hydrocephalus. Arch Neurol. 1989;46(6):608.

9. Brean A, Eide PK. Prevalence of probable idiopathic normal pressure hydrocephalus in a Norwegian population. Acta Neurol Scand. 2008;118(1):48-53.

10. Alvi MA, Brown D, Yolcu Y, et al. Prevalence and trends in management of idiopathic normal pressure hydrocephalus in the united states: insights from the national inpatient sample. World Neurosurg. 2020.

11. Mori E, Ishikawa M, Kato T, et al. Guidelines for management of idiopathic normal pressure hydrocephalus: second edition. Neurol Med Chir. 2012;52(11):775-809.

12. Hung AL, Vivas-Buitrago T, Adam A, et al. Ventriculoatrial versus ventriculoperitoneal shunt complications in idiopathic normal pressure hydrocephalus. Clin Neurol Neurosurg. 2017;157:1-6.

13. Louveau A, Smirnov I, Keyes TJ, et al. Structural and functional features of central nervous system lymphatic vessels. Nature. 2015;523(7560):337-341.

14. Absinta M, Ha SK, Nair G, et al. Human and nonhuman primate meninges harbor lymphatic vessels that can be visualized noninvasively by MRI. Elife. 2017;6.

15. Potokar M, Jorgacevski J, Zorec R. Astrocyte aquaporin dynamics in health and disease. Int J Mol Sci. 2016;17:7.

16. Nakada T. Virchow-Robin space and aquaporin-4: new insights on an old friend. Croat Med J. 2014;55(4):328-336.

17. Nakada T, Kwee IL. Fluid dynamics inside the brain barrier: current concept of interstitial flow, glymphatic flow, and cerebrospinal fluid circulation in the brain. Neuroscientist. 2019;25 (2):155-166.

18. Huber VJ, Igarashi H, Ueki S, Kwee IL, Nakada T. Aquaporin-4 facilitator TGN-073 promotes interstitial fluid circulation within the blood-brain barrier: [17O]H2O JJVCPE MRI study. Neuroreport. 2018;29(9):697-703.

19. Ikeshima-Kataoka H. Neuroimmunological implications of AQP4 in astrocytes. Int $J$ Mol Sci. 2016;17:8.

20. Desai B, Hsu Y, Schneller B, Hobbs JG, Mehta AI, Linninger A. Hydrocephalus: the role of cerebral aquaporin- 4 channels and computational modeling considerations of cerebrospinal fluid. Neurosurg Focus. 2016;41(3):E8.

21. Nakada T, Kwee IL, Igarashi H, Suzuki Y. Aquaporin-4 functionality and virchow-robin space water dynamics: physiological model for neurovascular coupling and glymphatic flow. Int J Mol Sci. 2017;18:8.

22. Rasmussen MK, Mestre H, Nedergaard M. The glymphatic pathway in neurological disorders. Lancet Neurol. 2018;17 (11):1016-1024.

23. Takizawa K, Matsumae M, Hayashi N, et al. The choroid plexus of the lateral ventricle as the origin of CSF pulsation is questionable. Neurol Med Chir. 2018;58(1):23-31.

24. Yamada S, Kelly E. Cerebrospinal fluid dynamics and the pathophysiology of hydrocephalus: new concepts. Seminars Iultrasound, CT MRI. 2016;37(2):84-91.

25. Eide PK, Ringstad G. Delayed clearance of cerebrospinal fluid tracer from entorhinal cortex in idiopathic normal pressure hydrocephalus: A glymphatic magnetic resonance imaging study. J Cereb Blood Flow Metab. 2019;39(7):1355-1368. 
26. Ringstad G, Valnes LM, Dale AM, et al. Brain-wide glymphatic enhancement and clearance in humans assessed with MRI. JCI Insight. 2018;3:13.

27. Ringstad G, Vatnehol SAS, Eide PK. Glymphatic MRI in idiopathic normal pressure hydrocephalus. Brain. 2017;140 (10):2691-2705.

28. Weller RO, Djuanda E, Yow HY, Carare RO. Lymphatic drainage of the brain and the pathophysiology of neurological disease. Acta Neuropathol. 2009;117(1):1-14.

29. Schley D, Carare-Nnadi R, Please CP, Perry VH, Weller RO. Mechanisms to explain the reverse perivascular transport of solutes out of the brain. J Theor Biol. 2006;238(4):962-974.

30. Brautigam K, Vakis A, Tsitsipanis C. Pathogenesis of idiopathic normal pressure hydrocephalus: A review of knowledge. J Clin Neurosci. 2019;61:10-13.

31. Qvarlander S, Lundkvist B, Koskinen LO, Malm J, Eklund A. Pulsatility in CSF dynamics: pathophysiology of idiopathic normal pressure hydrocephalus. J Neurol Neurosurg Psychiatry. 2013;84(7):735-741.

32. Matsumae M, Kuroda K, Yatsushiro S, et al. Changing the currently held concept of cerebrospinal fluid dynamics based on shared findings of cerebrospinal fluid motion in the cranial cavity using various types of magnetic resonance imaging techniques. Neurol Med Chir. 2019;59(4):133-146.

33. Bateman GA. The pathophysiology of idiopathic normal pressure hydrocephalus: cerebral ischemia or altered venous hemodynamics? AJNR Am J Neuroradiol. 2008;29(1):198-203.

34. Eide PK, Sorteberg W. Diagnostic intracranial pressure monitoring and surgical management in idiopathic normal pressure hydrocephalus: a 6-year review of 214 patients. Neurosurgery. 2010;66(1):80-91.

35. Skalicky P, Mladek A, Vlasak A, De Lacy P, Benes V, Bradac O. Normal pressure hydrocephalus-an overview of pathophysiological mechanisms and diagnostic procedures. Neurosurg Rev. 2019.

36. Lindstrom EK, Ringstad G, Mardal KA, Eide PK. Cerebrospinal fluid volumetric net flow rate and direction in idiopathic normal pressure hydrocephalus. Neuroimage Clin. 2018;20:731-741.

37. Eide PK, Hansson HA. Astrogliosis and impaired aquaporin-4 and dystrophin systems in idiopathic normal pressure hydrocephalus. Neuropathol Appl Neurobiol. 2018;44(5):474-490.

38. Hasan-Olive MM, Enger R, Hansson HA, Nagelhus EA, Eide PK. Loss of perivascular aquaporin-4 in idiopathic normal pressure hydrocephalus. Glia. 2019;67(1):91-100.

39. Ishikawa M, Oowaki H, Takezawa M, et al. Disproportionately enlarged subarachnoid space hydrocephalus in idiopathic normal-pressure hydrocephalus and its implication in pathogenesis. Acta Neurochir Suppl. 2016;122:287-290.

40. Leinonen V, Vanninen R, Rauramaa T. Cerebrospinal fluid circulation and hydrocephalus. Handb Clin Neurol. 2017;145:39-50.

41. Jones HC, Keep RF, Drewes LR. CNS fluid and solute movement: physiology, modelling and imaging. Fluids Barriers CNS. 2020;17:1.

42. Leal NS, Dentoni G, Schreiner B, et al. Alterations in mitochondria-endoplasmic reticulum connectivity in human brain biopsies from idiopathic normal pressure hydrocephalus patients. Acta Neuropathol Commun. 2018;6(1):102.

43. Hasan-Olive MM, Enger R, Hansson HA, Nagelhus EA, Eide PK. Pathological mitochondria in neurons and perivascular astrocytic endfeet of idiopathic normal pressure hydrocephalus patients. Fluids Barriers CNS. 2019;16(1):39.

44. Chistyakov AV, Hafner H, Sinai A, Kaplan B, Zaaroor M. Motor cortex disinhibition in normal-pressure hydrocephalus. $J$ Neurosurg. 2012;116(2):453-459.

45. Miyoshi N, Kazui H, Ogino A, et al. Association between cognitive impairment and gait disturbance in patients with idiopathic normal pressure hydrocephalus. Dement Geriatr Cogn Disord. 2005;20(2-3):71-76.
46. Nardone R, Golaszewski S, Schwenker K, et al. Cholinergic transmission is impaired in patients with idiopathic normal-pressure hydrocephalus: a TMS study. J Neural Transm. 2019;126(8):1073-1080.

47. Nikolakopoulou AM, Montagne A, Kisler K, et al. Pericyte loss leads to circulatory failure and pleiotrophin depletion causing neuron loss. Nat Neurosci. 2019;22(7):1089-1098.

48. Ueno M, Tomita S, Nakagawa T, et al. Effects of aging and HIF-1alpha deficiency on permeability of hippocampal vessels. Microsc Res Tech. 2006;69(1):29-35.

49. Andreone BJ, Chow BW, Tata A, et al. Blood-brain barrier permeability is regulated by lipid transport-dependent suppression of caveolae-mediated transcytosis. Neuron. 2017;94(3):581-594 e585.

50. Tripathi BJ, Tripathi RC. Vacuolar transcellular channels as a drainage pathway for cerebrospinal fluid. J Physiol. 1974;239 (1):195-206.

51. Eide PK, Hansson HA. Blood-brain barrier leakage of blood proteins in idiopathic normal pressure hydrocephalus. Brain Res. 2020;1727:146547.

52. Satow T, Aso T, Nishida S, et al. Alteration of venous drainage route in idiopathic normal pressure hydrocephalus and normal aging. Front Aging Neurosci. 2017;9:387.

53. Eidsvaag VA, Hansson HA, Heuser K, Nagelhus EA, Eide PK. Brain capillary ultrastructure in idiopathic normal pressure hydrocephalus: relationship with static and pulsatile intracranial pressure. J Neuropathol Exp Neurol. 2017;76(12):1034-1045.

54. Gautam J, Zhang X, Yao Y. The role of pericytic laminin in blood brain barrier integrity maintenance. Sci Rep. 2016;6:36450.

55. Isaacs AM, Williams MA, Hamilton MG. Current update on treatment strategies for idiopathic normal pressure hydrocephalus. Curr Treat Options Neurol. 2019;21:12.

56. Williams MA, Malm J. Diagnosis and Treatment of Idiopathic Normal Pressure Hydrocephalus. Continuum. 2016;22 (2Dementia):579-599.

57. Hasslacher-Arellano JF, Arellano-Aguilar G, Funes-Rodriguez JF, Lopez-Forcen S, Torres-Zapiain F, Dominguez-Carrillo LG. Ventriculo-gallbladder shunt: an alternative for the treatment of hydrocephalus. Cir Cir. 2016;84(3):225-229.

58. Arighi A, Di Cristofori A, Fenoglio C, et al. Cerebrospinal fluid level of aquaporin4: a new window on glymphatic system involvement in neurodegenerative disease? J Alzheimers Dis. 2019;69 (3):663-669

59. Williams MA, Relkin NR. Diagnosis and management of idiopathic normal-pressure hydrocephalus. Neurol Clin Pract. 2013;3 (5):375-385

60. Stolze H, Kuhtz-Buschbeck JP, Drucke H, et al. Gait analysis in idiopathic normal pressure hydrocephalus-which parameters respond to the CSF tap test? Clin Neurophysiol. 2000;111 (9):1678-1686.

61. Nikaido Y, Akisue T, Kajimoto Y, et al. Postural instability differences between idiopathic normal pressure hydrocephalus and Parkinson's disease. Clin Neurol Neurosurg. 2018;165:103-107.

62. Selge C, Schoeberl F, Zwergal A, et al. Gait analysis in PSP and NPH: dual-task conditions make the difference. Neurology. 2018;90(12):e1021-e1028.

63. Nikaido Y, Akisue T, Kajimoto Y, et al. The effect of CSF drainage on ambulatory center of mass movement in idiopathic normal pressure hydrocephalus. Gait Posture. 2018;63:5-9.

64. Nikaido Y, Urakami H, Akisue T, et al. Associations among falls, gait variability, and balance function in idiopathic normal pressure hydrocephalus. Clin Neurol Neurosurg. 2019;183:105385.

65. Gallagher R, Marquez J, Osmotherly P. Gait and balance measures can identify change from a cerebrospinal fluid tap test in idiopathic normal pressure hydrocephalus. Arch Phys Med Rehabil. 2018;99(11):2244-2250. 
66. Gallagher R, Marquez J, Osmotherly P. Clinimetric properties and minimal clinically important differences for a battery of gait, balance, and cognitive examinations for the tap test in idiopathic normal pressure hydrocephalus. Neurosurgery. 2019;84(6):E378E384.

67. Nikaido Y, Kajimoto Y, Akisue T, et al. Dynamic balance measurements can differentiate patients who fall from patients who do not fall in patients with idiopathic normal pressure hydrocephalus. Arch Phys Med Rehabil. 2019;100(8):1458-1466.

68. Isik AT, Kaya D, Ates Bulut E, Dokuzlar O, Soysal P. The outcomes of serial cerebrospinal fluid removal in elderly patients with idiopathic normal pressure hydrocephalus. Clin Interv Aging. 2019;14:2063-2069.

69. Liouta E, Gatzonis S, Kalamatianos T, et al. Finger tapping and verbal fluency post-tap test improvement in INPH: its value in differential diagnosis and shunt-treatment outcomes prognosis. Acta Neurochir. 2017;159(12):2301-2307.

70. Picascia M, Zangaglia R, Bernini S, Minafra B, Sinforiani E, Pacchetti C. A review of cognitive impairment and differential diagnosis in idiopathic normal pressure hydrocephalus. Funct Neurol. 2015;30(4):217-228.

71. Ko PW, Lee HW, Kang K. Frontal assessment battery and cerebrospinal fluid tap test in idiopathic normal-pressure hydrocephalus. Eur Neurol. 2017;77(5-6):327-332.

72. Larsson J, Israelsson H, Eklund A, Malm J. Epilepsy, headache, and abdominal pain after shunt surgery for idiopathic normal pressure hydrocephalus: the INPH-CRasH study. $J$ Neurosurg. 2018;128(6):1674-1683.

73. Matsuoka T, Kawano S, Fujimoto K, Kawahara M, Hashimoto H. Characteristics of cognitive function evaluation using the Montreal cognitive assessment in a cerebrospinal fluid tap test in patients with idiopathic normal pressure hydrocephalus. Clin Neurol Neurosurg. 2019;186:105524.

74. Sakakibara R, Kanda T, Sekido T, et al. Mechanism of bladder dysfunction in idiopathic normal pressure hydrocephalus. Neurourol Urodyn. 2008;27(6):507-510.

75. Halperin JJ, Kurlan R, Schwalb JM, Cusimano MD, Gronseth G, Gloss D. Practice guideline: idiopathic normal pressure hydrocephalus: response to shunting and predictors of response: report of the guideline development, dissemination, and implementation subcommittee of the american academy of neurology. Neurology. 2015;85(23):2063-2071.

76. Ferrari A, Milletti D, Giannini G, et al. The effects of cerebrospinal fluid tap-test on idiopathic normal pressure hydrocephalus: an inertial sensors based assessment. J Neuroeng Rehabil. 2020;17 (1):7.

77. Yamada S, Aoyagi Y, Yamamoto K, Ishikawa M. Quantitative evaluation of gait disturbance on an instrumented timed up-andgo test. Aging Dis. 2019;10(1):23-36.

78. Gallagher RM, Marquez J, Osmotherly P. Cognitive and upper limb symptom changes from a tap test in idiopathic normal pressure hydrocephalus. Clin Neurol Neurosurg. 2018;174:92-96.

79. Yamada S, Ishikawa M, Miyajima M, et al. Disease duration: the key to accurate CSF tap test in iNPH. Acta Neurol Scand. 2017;135(2):189-196.

80. Kaya D, Erken N, Ontan MS, Altun ZS, Isik AT. The applause sign in elderly patients with idiopathic normal pressure hydrocephalus. Appl Neuropsychol Adult. 2020;1-6.

81. Mahr CV, Dengl M, Nestler U, et al. Idiopathic normal pressure hydrocephalus: diagnostic and predictive value of clinical testing, lumbar drainage, and CSF dynamics. J Neurosurg. 2016;125 (3):591-597.

82. Kahlon B, Sundbarg G, Rehncrona S. Lumbar infusion test in normal pressure hydrocephalus. Acta Neurol Scand. 2005;111 (6):379-384
83. Raneri F, Zella MAS, Di Cristofori A, Zarino B, Pluderi M, Spagnoli D. Supplementary tests in idiopathic normal pressure hydrocephalus: a single-center experience with a combined lumbar infusion test and tap test. World Neurosurg. 2017;100:567-574

84. Ryding E, Kahlon B, Reinstrup P. Improved lumbar infusion test analysis for normal pressure hydrocephalus diagnosis. Brain Behav. 2018;8(11):e01125.

85. Schirinzi T, Sancesario GM, Di Lazzaro G, et al. Cerebrospinal fluid biomarkers profile of idiopathic normal pressure hydrocephalus. J Neural Transm. 2018;125(4):673-679.

86. Chen Z, Liu C, Zhang J, Relkin N, Xing Y, Li Y. Cerebrospinal fluid $\mathrm{A} \beta 42$, $\mathrm{t}$-tau, and $\mathrm{p}$-tau levels in the differential diagnosis of idiopathic normal-pressure hydrocephalus: a systematic review and meta-analysis. Fluids Barriers CNS. 2017;14:1.

87. Kim HJ, Lim TS, Lee SM, et al. Cerebrospinal fluid levels of beta-amyloid 40 and beta-amyloid 42 are proportionately decreased in amyloid positron-emission tomography negative idiopathic normal-pressure hydrocephalus patients. $J$ Clin Neurol. 2019;15(3):353-359.

88. Jeppsson A, Wikkelsö C, Blennow $\mathrm{K}$, et al. CSF biomarkers distinguish idiopathic normal pressure hydrocephalus from its mimics. J Neurol Neurosurg Psychiatry. 2019;90(10):1117-1123.

89. Abu-Rumeileh S, Giannini G, Polischi B, et al. Revisiting the cerebrospinal fluid biomarker profile in idiopathic normal pressure hydrocephalus: the bologna pro-hydro study. $J$ Alzheimer's Disease. 2019;68(2):723-733.

90. Jingami N, Uemura K, Asada-Utsugi M, et al. Two-point dynamic observation of alzheimer's disease cerebrospinal fluid biomarkers in idiopathic normal pressure hydrocephalus. $J$ Alzheimer's Disease. 2019;72(1):271-277.

91. Naganawa S, Taoka T. The glymphatic system: a review of the challenges in visualizing its structure and function with MR imaging. Magn Reson Med Sci. 2020.

92. Bae YJ, Choi BS, Kim JM, Choi JH, Cho SJ, Kim JH. Altered glymphatic system in idiopathic normal pressure hydrocephalus. Parkinsonism Relat Disord. 2020;82:56-60.

93. Yokota H, Vijayasarathi A, Cekic M, et al. Diagnostic performance of glymphatic system evaluation using diffusion tensor imaging in idiopathic normal pressure hydrocephalus and mimickers. Curr Gerontol Geriatr Res. 2019;2019:5675014.

94. Kamiya K, Hori M, Irie R, et al. Diffusion imaging of reversible and irreversible microstructural changes within the corticospinal tract in idiopathic normal pressure hydrocephalus. Neuroimage Clin. 2017;14:663-671.

95. Ghosh S, Lippa C. Diagnosis and prognosis in idiopathic normal pressure hydrocephalus. Am J Alzheimers Dis Other Demen. 2014;29(7):583-589.

96. Siasios I, Kapsalaki EZ, Fountas KN, et al. The role of diffusion tensor imaging and fractional anisotropy in the evaluation of patients with idiopathic normal pressure hydrocephalus: a literature review. Neurosurg Focus. 2016;41(3):E12.

97. Kockum K, Lilja-Lund O, Larsson EM, et al. The idiopathic normal-pressure hydrocephalus Radscale: a radiological scale for structured evaluation. Eur J Neurol. 2018;25(3):569-576.

98. Mantovani P, Albini-Riccioli L, Giannini G, et al. Anterior callosal angle: a new marker of idiopathic normal pressure hydrocephalus? World Neurosurg. 2020;139:e548-e552.

99. Jaraj D, Rabiei K, Marlow T, Jensen C, Skoog I, Wikkelso C. Estimated ventricle size using Evans index: reference values from a population-based sample. Eur J Neurol. 2017;24 (3):468-474.

100. Reeves BC, Karimy JK, Kundishora AJ, et al. Glymphatic system impairment in alzheimer's disease and idiopathic normal pressure hydrocephalus. Trends Mol Med. 2020;26(3):285-295. 
101. Shprecher D, Schwalb J, Kurlan R. Normal pressure hydrocephalus: diagnosis and treatment. Curr Neurol Neurosci Rep. 2008;8 (5):371-376.

102. He W, Fang X, Wang X, et al. A new index for assessing cerebral ventricular volume in idiopathic normal-pressure hydrocephalus: a comparison with Evans' index. Neuroradiology. 2020;62 (6):661-667.

103. Miyazaki K, Ishii K, Hanaoka K, Kaida H, Nakajima K. The tight medial and high convexity subarachnoid spaces is the first finding of idiopathic normal pressure hydrocephalus at the preclinical stage. Neurol Med Chir (Tokyo). 2019;59(11):436-443.

104. Akiba C, Gyanwali B, Villaraza S, et al. The prevalence and clinical associations of disproportionately enlarged subarachnoid space hydrocephalus (DESH), an imaging feature of idiopathic normal pressure hydrocephalus in community and memory clinic based Singaporean cohorts. J Neurol Sci. 2020;408:116510.

105. Neikter J, Agerskov S, Hellstrom P, et al. Ventricular volume is more strongly associated with clinical improvement than the evans index after shunting in idiopathic normal pressure hydrocephalus. AJNR Am J Neuroradiol. 2020;41(7):1187-1192.

106. Suehiro T, Kazui H, Kanemoto H, et al. Changes in brain morphology in patients in the preclinical stage of idiopathic normal pressure hydrocephalus. Psychogeriatrics. 2019;19(6):557-565.

107. Takagi K, Watahiki R, Machida T, Onouchi K, Kato K, Oshima M. Reliability and interobserver variability of evans' index and disproportionately enlarged subarachnoid space hydrocephalus as diagnostic criteria for idiopathic normal pressure hydrocephalus. Asian J Neurosurg. 2020;15:1.

108. Tinelli M, Guldemond N, Kehler U. Idiopathic Normal Pressure Hydrocephalus (iNPH): the cost-effectiveness of delivering timely and adequate treatment in Germany. Eur J Neurol. 2020.

109. Aimard G, Vighetto A, Gabet JY, Bret P, Henry E. Acetazolamide: an alternative to shunting in normal pressure hydrocephalus? Preliminary results. Rev Neurol. 1990;146 (6-7):437-439.

110. Swenson ER. Pharmacology of acute mountain sickness: old drugs and newer thinking. J Appl Physiol. 2016;120(2):204-215.

111. Alperin N, Oliu CJ, Bagci AM, et al. Low-dose acetazolamide reverses periventricular white matter hyperintensities in iNPH. Neurology. 2014;82(15):1347-1351.

112. Del Bigio MR, Di Curzio DL. Nonsurgical therapy for hydrocephalus: a comprehensive and critical review. Fluids Barriers CNS. 2016;13:3.

113. Gavrilov GV, Gaydar BV, Svistov DV, et al. Idiopathic normal pressure hydrocephalus (hakim-adams syndrome): clinical symptoms, diagnosis and treatment. Psychiatr Danub. 2019;31(Suppl 5):737-744.

114. Nakajima M, Miyajima M, Ogino I, et al. Preoperative phosphorylated tau concentration in the cerebrospinal fluid can predict cognitive function three years after shunt surgery in patients with idiopathic normal pressure hydrocephalus. $J$ Alzheimer's Disease. 2018;66(1):319-331.

115. Miyajima M, Kazui H, Mori E, Ishikawa M. SinphoniInvestigators obot. One-year outcome in patients with idiopathic normal-pressure hydrocephalus: comparison of lumboperitoneal shunt to ventriculoperitoneal shunt. J Neurosurg. 2016;125 (6):1483-1492.

116. Razay G, Wimmer M, Robertson I. Incidence, diagnostic criteria and outcome following ventriculoperitoneal shunting of idiopathic normal pressure hydrocephalus in a memory clinic population: a prospective observational cross-sectional and cohort study. BMJ Open. 2019;9(12):e28103.
117. Toma AK, Papadopoulos MC, Stapleton S, Kitchen ND, Watkins LD. Systematic review of the outcome of shunt surgery in idiopathic normal-pressure hydrocephalus. Acta Neurochir. 2013;155(10):1977-1980.

118. Bådagård $\mathrm{H}$, Braun $\mathrm{M}$, Nilsson $\mathrm{D}$, Stridh L, Virhammar $\mathrm{J}$. Negative predictors of shunt surgery outcome in normal pressure hydrocephalus. Acta Neurol Scand. 2019;141(3):219-225.

119. Feletti A, d'Avella D, Wikkelso C, et al. Ventriculoperitoneal shunt complications in the european idiopathic normal pressure hydrocephalus multicenter study. Oper Neurosurg. 2019;17 (1):97-102.

120. Farahmand D, Saehle T, Eide PK, Tisell M, Hellstrom P, Wikkelso C. A double-blind randomized trial on the clinical effect of different shunt valve settings in idiopathic normal pressure hydrocephalus. J Neurosurg. 2016;124(2):359-367.

121. Giordan E, Palandri G, Lanzino G, Murad MH, Elder BD. Outcomes and complications of different surgical treatments for idiopathic normal pressure hydrocephalus: a systematic review and meta-analysis. J Neurosurg. 2018;1-13.

122. Liu A, Sankey EW, Jusue-Torres I, et al. Clinical outcomes after ventriculoatrial shunting for idiopathic normal pressure hydrocephalus. Clin Neurol Neurosurg. 2016;143:34-38.

123. Krahulik D, Vaverka M, Hrabalek L, et al. Ventriculoperitoneal shunt in treating of idiopathic normal pressure hydrocephalus-single-center study. Acta Neurochir. 2020;162 (1):1-7.

124. Clark DJ, Chakraborty A, Roebuck DJ, Thompson DN. Ultrasound guided placement of the distal catheter in paediatric ventriculoatrial shunts-an appraisal of efficacy and complications. Childs Nerv Syst. 2016;32(7):1219-1225.

125. Nunoo-Mensah JW, Rosen M, Chan LS, Wasserberg N, Beart RW. Prevalence of intra-abdominal surgery: what is an individual's lifetime risk? South Med J. 2009;102(1):25-29.

126. McGovern RA, Kelly KM, Chan AK, Morrissey NJ, McKhann GM 2nd. Should ventriculoatrial shunting be the procedure of choice for normal-pressure hydrocephalus? J Neurosurg. 2014;120(6):1458-1464.

127. Nakajima M, Miyajima M, Ogino I, et al. Shunt intervention for possible idiopathic normal pressure hydrocephalus improves patient outcomes: a nationwide hospital-based survey in Japan. Front Neurol. 2018;9.

128. Kazui H, Miyajima M, Mori E, Ishikawa M, Investigators S. Lumboperitoneal shunt surgery for idiopathic normal pressure hydrocephalus (SINPHONI-2): an open-label randomised trial. Lancet Neurol. 2015;14(6):585-594.

129. Oliveira MF, Teixeira MJ, Reis RC, Petitto CE, Gomes Pinto FC. Failed ventriculoperitoneal shunt: is retrograde ventriculosinus shunt a reliable option? World Neurosurg. 2016;92:445-453.

130. Paidakakos N, Borgarello S, Naddeo M. Indications for endoscopic third ventriculostomy in normal pressure hydrocephalus. Acta Neurochir Suppl. 2012;113:123-127.

131. Pinto FC, Saad F, Oliveira MF, et al. Role of endoscopic third ventriculostomy and ventriculoperitoneal shunt in idiopathic normal pressure hydrocephalus: preliminary results of a randomized clinical trial. Neurosurgery. 2013;72(5):845-853; discussion 853-844.

132. Hailong F, Guangfu H, Haibin T, et al. Endoscopic third ventriculostomy in the management of communicating hydrocephalus: a preliminary study. $J$ Neurosurg. 2008;109(5):923-930.

133. Tudor KI, Tudor M, McCleery J, Car J. Endoscopic third ventriculostomy (ETV) for idiopathic normal pressure hydrocephalus (iNPH). Cochrane Database Syst Rev. 2015;(7):CD010033. 


\section{Publish your work in this journal}

Clinical Interventions in Aging is an international, peer-reviewed journal focusing on evidence-based reports on the value or lack thereof of treatments intended to prevent or delay the onset of maladaptive correlates of aging in human beings. This journal is indexed on PubMed Central, MedLine, CAS, Scopus and the Elsevie
Bibliographic databases. The manuscript management system is completely online and includes a very quick and fair peer-review system, which is all easy to use. Visit http://www.dovepress.com/ testimonials.php to read real quotes from published authors.

Submit your manuscript here: https://www.dovepress.com/clinical-interventions-in-aging-journal 